\title{
Excitatory amino acid binding sites in the trigeminal principal sensory and spinal trigeminal nuclei of the rat
}

\author{
Sara J. Tallaksen-Greene ${ }^{\mathrm{a}, \mathrm{b}}$, Anne B. Young ${ }^{\mathrm{b}}$, John B. Penney ${ }^{\mathrm{b}}$ and Alvin J. Beitz \\ ${ }^{a}$ Department of Veterinary Biology, University of Minnesota, St. Paul, MN (USA) and ${ }^{b}$ Department of Neurology, University of Michigan, \\ Ann Arbor, MI 48104-1687 (USA)
}

(Received 17 January 1992; Revised version received 27 March 1992; Accepted 30 March 1992)

Key words: Glutamate; Autoradiography; NMDA; AMPA; Kainate; Metabotropic receptor; Trigeminal nucleus; Nociception

\begin{abstract}
Quantitative autoradiography was used to examine the density and distribution of excitatory amino acid (EAA) binding site subtypes in the principal sensory and spinal trigeminal nuclei of the rat trigeminal complex. The highest densities of $N$-methyl-D-aspartate (NMDA), $\alpha$-amino-3hydroxy-5-methylisoxazole-4-proprionic acid (AMPA), kainate and metabotropic receptors were found in the superficial laminae (I and II) of subnucleus caudalis, a region known to be densely innervated by primary afferent nociceptive terminals. Lower densities of EAA binding sites were observed in spinal subnuclei interpolaris and oralis and within the principal sensory nucleus. These results are consistent with the hypothesis that EAAs are involved in primary afferent nociceptive neurotransmission.
\end{abstract}

Recent studies suggest that nociception in the trigeminal nuclear complex may be mediated by excitatory amino acid (EAA) neurotransmitters. Immunohistochemical studies have identified EAAs in neuronal perikarya $[13-15,28]$, nerve fibers $[14,28]$ and synaptic terminals $[5,28]$ within the spinal trigeminal nucleus. In addition, EAAs have been identified in trigeminal primary sensory neurons [27]. Similar findings have been reported in the spinal cord dorsal horn [7], a region structurally and functionally homologous to the spinal trigeminal nucleus caudalis (medullary dorsal horn). Release of EAAs has been reported in response to noxious stimulation $[20,23]$ and following infusion of either veratridine (a sodium channel activator) $[2,23]$ or substance $P$ [24]. Physiological studies have demonstrated that EAAs elicit excitatory responses from neurons in the superficial laminae (I and II) of both the spinal [1, 6, 21] and medullary dorsal horn [19]. Receptors mediating the effects of EAAs have been classified into pharmacologically distinct subtypes; $N$-methyl-D-aspartate (NMDA), $\alpha$-amino-3-hydroxy-5-methylisoxazole-4-proprionic acid (AMPA), kainate (KA) and metabotropic receptors [29]. EAA binding sites in the trigeminal nucleus have been previously reported [11], although no comparison of

Correspondence: S.J. Tallaksen-Greene, Neuroscience Lab. Building, 1103 E. Huron, Ann Arbor, MI 48104-1687, USA.
EAA receptor subtypes was made. The present study was designed to determine the relative density and distribution of EAA binding site subtypes in the regions of the trigeminal nucleus known to receive nociceptive primary afferent fibers: the spinal trigeminal nucleus and the principal sensory nucleus of the trigeminal nerve.

Sixteen male Sprague-Dawley rats (175-225 g; Spar$\tan$ Labs, Hastings, MI) were used in this study. Autoradiographic assays for NMDA, AMPA, metabotropic and kainate binding sites were performed using previously described techniques $[4,29]$. Briefly, sections were prewashed in ice-cold incubation buffer for $30 \mathrm{~min}$, dried under a stream of cool air and then immersed in incubation buffer containing tritiated ligand and the appropriate blocking agents $\left(45 \mathrm{~min}, 4^{\circ} \mathrm{C}\right)$. NMDA binding was assayed with $65 \mathrm{nM} \mathrm{L}-\left[{ }^{3} \mathrm{H}\right]$ glutamate (specific activity = $56 \mathrm{Ci} / \mathrm{mmol}$ ) in $50 \mathrm{mM}$ Tris-acetate, $1.0 \mu \mathrm{M} \mathrm{KA}, 2.5 \mu \mathrm{M}$ quisqualic acid (QA) (pH 7.2). Non-specific binding was determined by adding $1 \mathrm{mM}$ NMDA to the ligand buffer. AMPA receptors were measured with 15-36 nM $\mathrm{DL}-\left[{ }^{3} \mathrm{H}\right] \alpha$-amino-3-hydroxy-5-methylisoxazole-4-proprionate (AMPA; spec. act. $=60 \mathrm{Ci} / \mathrm{mmol}$ ) in $50 \mathrm{mM}$ Tris$\mathrm{HCl}, 2.5 \mathrm{mM} \mathrm{CaCl}_{2}, 30 \mathrm{mM} \mathrm{KSCN}(\mathrm{pH}$ 7.2). Glutamate $(1 \mathrm{mM})$ was added to the ligand buffer to determine nonspecific binding. Metabotropic binding sites were assessed with $100 \mathrm{nM} \mathrm{L-}\left[{ }^{3} \mathrm{H}\right]$ glutamate (spec. act. $=45 \mathrm{Ci} /$ $\mathrm{mmol}$ ) in $50 \mathrm{mM}$ Tris- $\mathrm{HCl}, 2.5 \mathrm{mM} \mathrm{CaCl}, 30 \mathrm{mM}$ $\mathrm{KSCN}, 100 \mu \mathrm{M}$ NMDA, $10 \mu \mathrm{M}$ AMPA (pH 7.2). Non- 
specific binding was determined with the addition of 2.5 $\mu \mathrm{M}$ QA to the ligand buffer. KA binding sites were assayed using $60 \mathrm{nM}\left[{ }^{3} \mathrm{H}\right] \mathrm{KA}$ (spec. act. $=7.8 \mathrm{Ci} / \mathrm{mmol}$ ) in $50 \mathrm{mM}$ Tris-acetate buffer ( $\mathrm{pH}$ 7.2). Non-specific binding was measured with $100 \mu \mathrm{M} \mathrm{KA}$ added to the ligand buffer. Controls for regional tritium quenching were included using $65 \mathrm{nM}\left[{ }^{14} \mathrm{C}\right]$ glutamate (spec. act. $=50 \mathrm{mCi} /$ $\mathrm{mmol})$ in $50 \mathrm{mM}$ Tris-acetate $(\mathrm{pH} \mathrm{7.4)}$ to assess total glutamate binding. Non-specific binding was determined with the addition of $1 \mathrm{mM}$ glutamate to the ligand buffer. In all experiments, the binding reaction was terminated by rapidly rinsing each slide with 4 squirts of ice-cold buffer followed by 2 squirts of $2.5 \%$ glutaraldehyde in acetone. The sections were dried under a stream of hot air, apposed to tritium-sensitive film (Hyperfilm, Amersham) and exposed for $2-8\left({ }^{3} \mathrm{H}\right)$ or $16\left({ }^{14} \mathrm{C}\right)$ weeks with known radioactive standards. Autoradiograms were analyzed using a computer-assisted densitometry program (MCID system, Imaging Research, St. Catharine's Ont., Canada). For each area, triplicate sections were analyzed from a minimum of three animals. For each ligand, regional binding densities were compared using a one factor ANOVA followed by Scheffé posthoc tests. A reference area of known high binding was included for each ligand as an index of the relative level of each binding site. The reference region for NMDA and KA binding sites was the granule cell layer of the cerebellum and for
AMPA and metabotropic binding sites the molecular layer of the cerebellar cortex was used.

There was a heterogeneous distribution of all subtypes of excitatory amino acid binding sites within the spinal trigeminal nucleus (Fig. 1) and principal sensory nucleus of the trigeminal nerve. Regional differences in EAA binding site subtypes are summarized in Table $\mathrm{I}$, in terms of both the absolute amount of ligand bound and the amount bound relative to the cerebellar cortex. The highest density of ligand binding for all EAA subtypes was observed in the marginal zone (lamina I) and substantia gelatinosa (lamina II) of the spinal trigeminal nucleus caudalis (Table I, Fig. 1). Within this region, AMPA binding sites had the highest relative density followed by metabotropic and KA receptor subtypes; NMDA receptors exhibited the lowest relative density. The density of each binding site subtype within the inner magnocellular layer of nucleus caudalis (laminae III and IV) was significantly reduced compared to binding in the outer-most laminae of the nucleus. Within this region, there was a slightly higher relative density of metabotropic binding sites compared to AMPA and KA binding sites; again, NMDA binding sites had the lowest relative density. In spinal trigeminal nucleus interpolaris, the density of metabotropic, kainate and NMDA binding sites was lower than that seen in laminae III and IV of nucleus caudalis; whereas AMPA binding was slightly higher,

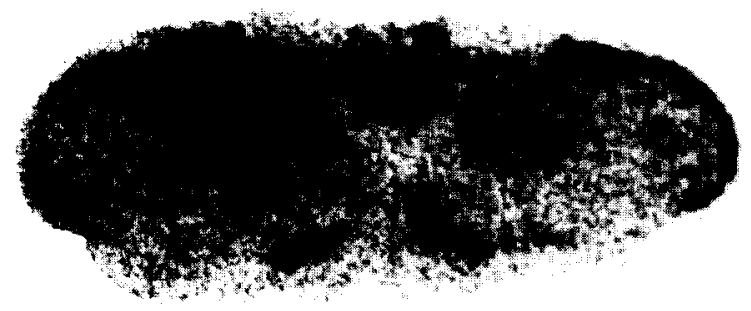

C

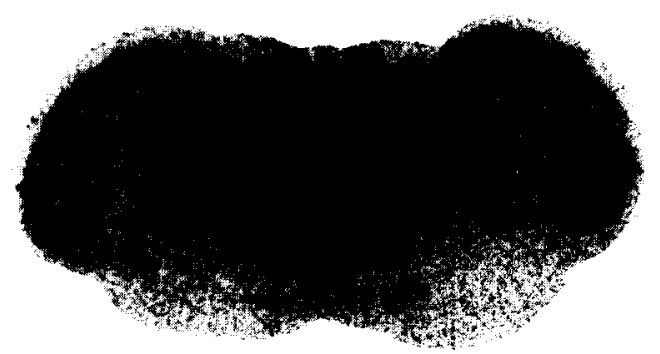

D

\section{$\boldsymbol{E}$}

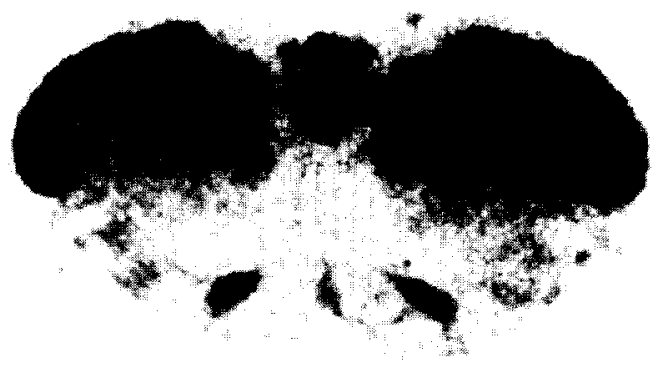

Fig. 1. Autoradiographs showing excitatory amino acid binding sites in rat spinal trigeminal subnucleus caudalis (Sp5C). A: $\left[{ }^{3} \mathrm{H}\right] \mathrm{AMPA}$ binding sites. B: $\left[{ }^{3} \mathrm{H}\right]$ glutamate (metabotropic) binding sites. C: NMDA-sensitive $\left[{ }^{3} \mathrm{H}\right]$ glutamate binding sites. D: $\left[{ }^{3} \mathrm{H}\right]$ kainate binding sites. Highest density of binding is seen in the superficial laminae ( $\mathrm{I}$ and II). 
TABLE I

DENSITY OF EXCITATORY AMINO ACID BINDING SITES IN SPINAL TRIGEMINAL NUCLEUS (Sp5) AND PRINCIPAL SENSORY NUCLEUS OF THE TRIGEMINAL NERVE.

\begin{tabular}{|c|c|c|c|c|c|c|c|c|}
\hline \multicolumn{9}{|l|}{ Sp5 subdivision: } \\
\hline Laminae I and II & $110 \pm 13$ & $(27 \%)$ & $1102 \pm 79$ & $(113 \%)$ & $772 \pm 40$ & $(66 \%)$ & $660 \pm 28$ & $(76 \%)$ \\
\hline Laminae III and IV & $30 \pm 08^{*}$ & $(7 \%)$ & $229 \pm 25^{*}$ & $(23 \%)$ & $315 \pm 28^{*}$ & $(27 \%)$ & $217 \pm 16^{*}$ & $(25 \%)$ \\
\hline Interpolaris & $19 \pm 09^{*}$ & $(5 \%)$ & $247 \pm 37^{*}$ & $(25 \%)$ & $255 \pm 15^{*}$ & $(22 \%)$ & $124 \pm 27^{*}$ & $(14 \%)$ \\
\hline
\end{tabular}

Data represent the mean specific binding in fmol/mg protein \pm S.E.M. NMDA, $N$-methyl-D-aspartate binding sites; AMPA, $\alpha$-amino-3-hydroxy-5methylisoxazole-4-proprionate binding sites; METAB, metabotropic binding sites; KA, kainic acid binding sites. \%, represents the percent of binding compared to a reference area of known high ligand binding (see text for details). This value provides an index of the relative level of each binding site compared to other regions of the central nervous system. N.S., no significant binding.

* Significantly different from laminae I and II $(P \leq 0.01)$; $\$$ significantly different from Interpolaris $(P \leq 0.05)$.

due largely to patches of high density binding in the rostral portion of the nucleus. Low levels of AMPA, metabotropic and KA binding sites were detected in spinal trigeminal nucleus oralis; NMDA binding in this region did not exceed background levels. The density and distribution of binding sites in the principal sensory nucleus of the trigeminal complex was very similar to, although slightly lower than, binding densities in the magnocellular layer of nucleus caudalis.

Our results indicate that the greatest proportion of EAA binding sites in the trigeminal nuclear complex are located in the superficial laminae of nucleus caudalis. These findings are consistent with previous studies reporting moderate densities of total [11] and NMDA-sensitive [17] L- $\left[{ }^{3} \mathrm{H}\right]$ glutamate binding in the substantia gelatinosa of the medullary (trigeminal nucleus caudalis) and cervical dorsal horn. In addition, our results are supported by studies in spinal cord that have shown that the highest densities of NMDA, kainate and AMPA binding sites are in laminae I and II of several species [12, 16, 17]. In the present study, we have observed remarkable similarities in the distribution of EAA binding subtypes within the trigeminal nuclear complex, much like the pattern reported in spinal cord $[12,16]$. All four EAA receptor subtypes showed highest densities in the superficial laminae of nucleus caudalis and, except for AMPA, the lowest densities in nucleus oralis. Although the use of single ligand concentrations does not allow for true comparison of the total density $\left(B_{\max }\right)$ of binding site subtypes, the relative density of EAA receptor subtypes in the trigeminal nuclear complex suggests that NMDA receptors are the least prevalent. The significance of this finding is unclear; further studies are needed to establish the relative importance of EAA receptor subtypes in trigeminal nociception.

Current evidence suggests that EAAs mediate synaptic transmission at the central terminals of primary afferent neurons. Early biochemical reports implicating EAAs as primary afferent neurotransmitters [18] have been supported by physiological $[1,21]$ and pharmacological $[8$, 20] data and by immunohistochemical studies that have identified EAAs in primary afferent cell bodies and nerve terminals $[5,7,26,27]$. In addition to the role EAAs are likely to play as neurotransmitters of primary sensory fibers, there is evidence to suggest that EAAs are involved in other aspects of primary afferent neurotransmission. For example, a non-NMDA EAA receptor has been implicated in presynaptic inhibition of primary afferent fibers [10]. Our observations of high densities of EAA receptors in laminae I and II of nucleus caudalis, as compared to other regions of the trigeminal nuclear complex, best support the notion that EAAs mediate sensory neurotransmission at the level of the primary afferent terminal. Furthermore, since the majority of input to laminae I and II is small myelinated (A $\delta$ ) and unmyelinated (C) fibers of nociceptive and thermal receptors [25] the high density of EAA binding sites in this region is consistent with the hypothesis that EAA receptors in the trigeminal nucleus are involved in the processing of nociceptive information.

Pharmacological studies have shown that nociceptive neurotransmission is facilitated by EAA agonists [1,9] and reduced by EAA antagonists $[8,20]$ supporting the hypothesis that nociception is mediated by EAA transmitters. In addition, both peripheral noxious stimulation and local substance $P$ infusion [24] have been reported to 
increase the concentration of extracellular EAAs in spinal cord dorsal horn [23]. Recently, Aanonsen et al. [1] have shown that nociceptive neurons in the spinal cord are selectively activated by NMDA, quisqualate and AMPA. Within the trigeminal system, Salt and Hill [20] originally proposed that EAA receptors are involved in nociception based on the observation that a broad-spectrum EAA antagonist reduced the response of nucleus caudalis neurons to noxious stimuli. Recently, a glutaminergic primary afferent pathway projecting from the tooth pulp to trigeminal nucleus caudalis has been described by Clements et al. [5]. It is likely that these fibers are involved in pain transmission, since dental pulp is believed to be predominantly nociceptive in function [22]. In addition to a sensory function, EAAs also appear to be involved in autonomic and endocrine responses to pain. Bereiter and Gann [3] have shown that microinjection of glutamate into the superficial laminae of the spinal trigeminal nucleus increases arterial pressure and plasma ACTH concentrations [3]. The identification of glutamate immunoreactivity in both trigeminothalamic neurons [13,15] and trigeminal interneurons [13] suggests that trigeminal second-order neurons may utilize EAA neurotransmitters as well. Taken together, these data underscore the complexity of the involvement of EAAs in nociception. The extent to which the EAA receptor subtypes mediate different physiological events is unknown. However, the high density of all subtypes we observed in the superficial laminae of nucleus caudalis supports a role for EAAs in trigeminal nociception. The overlapping distributions of EAA receptor subtypes that we observed in the trigeminal nuclear complex may further reflect subtype mediation of different aspects of trigeminal nociception.

Supported by NIH Awards DE06682, DA06687 and DC01086 to A.J.B.

1 Aanonsen, L.M., Lei, S. and Wilcox, G.L., Excitatory amino acid receptors and nociceptive neurotransmission in rat spinal cord, Pain, 41 (1990) 309-321.

2 Beitz, A.J. and Mullett, M.A., A comparison of amino acids in four brainstem nuclei using in vivo microdialysis, Current Separations (Abstr.), 10 (1991) 82.

3 Bereiter, D.A. and Gann, D.S. Glutamate activation of neurons within trigeminal nucleus caudalis increases adrenocorticotropin in the cat, Pain, 33 (1988) 341-348.

4 Cha, J.J., Makoweic, R.L., Penney, J.B. and Young, A.B., L$\left[{ }^{3} \mathrm{H}\right]$ Glutamate labels the metabotropic excitatory amino acid receptor in rodent brain, Neurosci. Lett., 113 (1990) 78-83.

5 Clements, J.R., Magnusson, K.R., Hautman, J. and Beitz, A.J., Rat tooth pulp projections to spinal trigeminal subnucleus caudalis are glutamate-like immunoreactive, J. Comp. Neurol., 15 (1991) $2-9$.

6 Curtis, D.R., Phillis, J.W. and Watkins, J.C., The chemical excita- tion of spinal neurons by certain acidic amino acids, J. Physiol., 150 (1960) 656-682.

7 De Biasi, S. and Rustioni, A., Glutamate and substance P coexist in primary afferent terminals in the superficial laminae of spinal cord, Proc. Natl. Acad. Sci. USA, 85 (1988) 7820-7824.

8 Dickenson, A.H. and Aydar, E., Antagonism at the glycine site on the NMDA receptor reduces spinal nociception in the rat, Neurosci. Lett., 121 (1991) 263-266.

9 Dougherty, P.M. and Willis, W.D., Modification of the responses of primate spinothalamic neurons to mechanical stimulation by excitatory amino acids and an $N$-methyl-D-aspartate antagonist,Brain Res., 542 (1991) 15-22.

10 Evans, R.H. and Long, S.K., Primary afferent depolarization in the rat spinal cord is mediated by pathways utilizing NMDA and nonNMDA receptors, Neurosci. Lett., 100 (1989) 231-236.

11 Greenamyre, J.T., Young, A.B. and Penney, J.B., Quantitative autoradiographic distribution of $\mathrm{L}-\left[{ }^{3} \mathrm{H}\right]$ glutamate-binding sites in rat central nervous system, J. Neurosci., 4 (1984) 2133-2144.

12 Jansen, K.L.R., Faull, R.L.M., Dragunow, M. and Waldvogel, H., Autoradiographic localisation of NMDA, quisqualate, and kainic acid receptors in human spinal cord, Neurosci. Lett., 108 (1990) 53-57.

13 Madl, J.E., Beitz, A.J., Johnson, R.L. and Larson, A.A., Monoclonal antibodies specific for fixative-modified aspartate: immunohistochemical localization in the rat CNS, J. Neurosci., 7 (1987) 26392650.

14 Magnusson, K.R., Clements, J.R., Larson, A.A., Madl, J.E. and Beitz, A.J., Co-localization of fixative-modified glutamate and glutaminase in neurons of the spinal trigeminal nucleus of the rat: an immunohistochemical and immunoradiochemical analysis, J. Comp. Neurol., 247 (1986) 477-490.

15 Magnusson, K.R., Clements, J.R., Larson, A.A., Madl, J.E. and Beitz, A.J., Localization of glutamate in trigemino-thalamic projection neurons: A combined retrograde transport-immunohistochemical study, Somatosens. Res., 4 (1987) 177-190.

16 Mitchell, J.J. and Anderson, K.J., Quantitative autoradiographic analysis of excitatory amino acid receptors in the cat spinal cord, Neurosci. Lett., 124 (1991) 269-272.

17 Monaghan, D.T. and Cotman, C.W., Distribution of $N$-methyl-Daspartate-sensitive $\mathrm{L}-\left[{ }^{3} \mathrm{H}\right]$ glutamate-binding sites in rat brain, $\mathrm{J}$. Neurosci., 5 (1985) 2909-2919.

18 Potashner, S.J. and Tran, P.L., Decreased uptake and release of D-aspartate in the guinea pig spinal cord after dorsal root section, J. Neurochem., 42 (1984) 1135-1144.

19 Salt, T.E. and Hill, R.G., Excitatory amino acids as transmitter candidates of vibrissae afferent fibres to the rat trigeminal nucleus caudalis, Neurosci. Lett., 22 (1981) 183-187.

20 Salt, T.E. and Hill, R.G., Pharmacological differentiation between responses of rat medullary dorsal horn neurons to noxious mechanical and noxious thermal cutaneous stimuli, Brain Res., 263 (1983) $167-171$

21 Schneider, S.P. and Perl, E.R., Comparison of primary afferent and glutamate excitation of neurons in the mammalian spinal dorsal horn, J. Neurosci., 8 (1988) 2062-2073.

22 Sessle, B.J., Dental deafferentation can lead to the development of chronic pain. In I. Klineberg and B.J. Sessle (Eds.), Oro-Facial Pain and Neuromuscular Dysfunction. Mechanisms and Clinical Correlates, Pergamon, Oxford, 1987, pp. 115-129.

23 Skilling, S.R., Smullin, D.H., Beitz, A.J. and Larson, A.A., Extracellular amino acid concentrations in the dorsal spinal cord of freely moving rats following veratridine and nociceptive stimulation, J. Neurochem., 51 (1988) 127-132. 
24 Smullin, D.H., Skilling, S.R. and Larson, A.A., Interactions between substance-P, calcitonin gene-related peptide, taurine and excitatory amino acids in the spinal cord, Pain, 42 (1990) 93-101.

25 Sugiura, Y., Lee, C.L. and Perl, E.R., Central projections of identified, unmyelinated $(C)$ afferent fibers innervating mammalian skin, Science, 234 (1986) 358-361.

26 Tracey, D.J., De Biasi, S., Phend, K. and Rustioni, A., Aspartatelike immunoreactivitiy in primary afferent neurons. Neuroscience, 40 (1991) 673-686.

27 Wanaka, A., Shiotani, Y., Kiyama, H., Matsuyama, T., Kamada, T., Shiosaka, S. and Tohyama, M., Glutamate-like immunoreactive structures in primary sensory neurons in the rat detected by specific antiserum against glutamate, Exp. Brain Res., 65 (1987) 691-694.

28 Weinberg, R.J., Conti, F., Van Eyck, S.L., Petrusz, P. and Rustioni, A., Glutamate immunoreactivity in the superficial laminae of rat dorsal horn and spinal trigeminal nucleus. In T.P. Hicks, D. Lodge and $\mathrm{H}$. McLennan (Eds.), Excitatory Amino Acid Transmission, Neurology and Neurobiology, Vol. 24, Liss, New York, 1987, pp. 126-133.

29 Young, A.B. and Fagg, G.E., Excitatory amino acid receptors in the brain: membrane binding and receptor autoradiographic approaches, TIPS, 11 (1990) 126-133. 